УДК 517.54

\author{
A. K. Bakhtin, I. V. Denega
}

\title{
WEAKENED PROBLEM ON EXTREMAL DECOMPOSITION OF THE COMPLEX PLANE
}

A. K. Bakhtin, I. V. Denega. Weakened problem on extremal decomposition of the complex plane, Mat. Stud. 51 (2019), 35-40.

The paper deals with the problem of the maximum of the functional

$$
r^{\gamma}\left(B_{0}, 0\right) \prod_{k=1}^{n} r\left(B_{k}, a_{k}\right)
$$

where $B_{0}, \ldots, B_{n}, n \geqslant 2$, are pairwise disjoint domains in $\overline{\mathbb{C}}, a_{0}=0,\left|a_{k}\right|=1, k \in\{1, \ldots, n\}$ and $\gamma \in(0, n](r(B, a)$ is the inner radius of the domain $B \subset \overline{\mathbb{C}}$ with respect to $a)$. We show that the functional attains its maximum at a configuration of the domains $B_{k}$ and the points $a_{k}$ possessing rotational $n$-symmetry. The proof is due to Dubinin [1] for $\gamma=1$ and to Kuz'mina [3] for $0<\gamma<1$. Subsequently, Kovalev [4] solved this problem for $n \geqslant 5$ under the additional assumption that the angles between neighbouring line segments $\left[0, a_{k}\right]$ do not exceed $2 \pi / \sqrt{\gamma}$. In the paper, we obtain some estimate of the functional for $\gamma \in(1, n]$.

In the geometric function theory of complex variable extremal problems on non-overlapping domains are well-known classic direction. A lot of such problems are reduced to determination of the maximum of the product of inner radii on the system of non-overlapping domains satisfying certain conditions (see, for example, [1-18]). Let $\mathbb{C}$ be the complex plane, $\overline{\mathbb{C}}=\mathbb{C} \cup\{\infty\}$ be a point compactification, $\mathbb{N}, \mathbb{R}$ be the sets of natural and real numbers, respectively, and $\mathbb{R}^{+}=(0, \infty)$. Let $r(B, a)$ be the inner radius of the domain $B \subset \overline{\mathbb{C}}$ relative to a point $a \in B$.

The system of points $A_{n}:=\left\{a_{k} \in \mathbb{C}, k=\overline{1, n}\right\}, n \in \mathbb{N}, n \geqslant 2$ is called $n$-radial, if $\left|a_{k}\right| \in \mathbb{R}^{+}$for $k=\overline{1, n}$ and $0=\arg a_{1}<\arg a_{2}<\ldots<\arg a_{n}<2 \pi$. Denote $a_{n+1}:=a_{1}$, $\alpha_{k}:=\frac{1}{\pi} \arg \frac{a_{k+1}}{a_{k}}, \alpha_{n+1}:=\alpha_{1}, k=\overline{1, n}, \sum_{k=1}^{n} \alpha_{k}=2$.

Let us consider an open extremal problem which was formulated in [1] in the list of unsolved problems. Later, it was repeated in monograph [8].

Problem. For any fixed value of $\gamma \in(0, n]$ to find the maximum of the functional

$$
I_{n}(\gamma)=r^{\gamma}\left(B_{0}, 0\right) \prod_{k=1}^{n} r\left(B_{k}, a_{k}\right)
$$

2010 Mathematics Subject Classification: 30C75.

Keywords: extremal problems with free poles; inner radius of domain; non-overlapping domains; complex plane; transfinite diameter; theorem on minimizing of the area; Cauchy inequality.

doi:10.15330/ms.51.1.35-40

(C) A. K. Bakhtin, I. V. Denega, 2019 
where $B_{0}, B_{1}, B_{2}, \ldots, B_{n}, n \geqslant 2$, are mutually non-overlapping domains, $a_{0}=0,\left|a_{k}\right|=1$, $a_{k} \in B_{k} \subset \overline{\mathbb{C}}, k=\overline{1, n}$.

Nowadays, this task is not completely solved, its solutions for are only known certain particular cases (see, for example, [1-17]). In [1,2] the problem was solved for any $n \geqslant 2$ and $\gamma=1$. In $1996 \mathrm{~L}$. V. Kovalev [4] got the solution of this problem with some restrictions on the location of points on the unit circle and, namely, for $n \geqslant 5$ and the subclass of point systems satisfying the following condition

$$
0<\alpha_{k} \leqslant 2 / \sqrt{\gamma}, k=\overline{1, n} .
$$

It is clear that these conditions are sufficiently stringent conditions. They significantly narrow the set of feasible configurations. In 2003, in paper of G.V. Kuz'mina [3] in the case of simply connected domains this problem was also been studied for $\gamma \in(0,1]$ by another method. In 2008, in the monograph [9, p. 255] it was shown that the result of V.N. Dubinin holds for an arbitrary $\gamma \in \mathbb{R}^{+}$and some number $n_{0}(\gamma)$. The problem was solved in [12] for $n \geqslant 8$ and $1<\gamma \leqslant \sqrt[4]{n}$, and in [13] for $n \geqslant 12$ and $1<\gamma \leqslant n^{0,45}$.

It should be noted that in the case $\gamma>1$ the method developed in paper of V.N. Dubinin [1] can not be applied. We note that the maximum of the functional $I_{n}(\gamma)$ was studying in papers $[1,2,4,8,9,12,13]$. In particular cases for certain values of $\gamma$ it is shown that the following inequality holds

$$
I_{n}(\gamma) \leqslant\left(\frac{4}{n}\right)^{n} \frac{\left(\frac{4 \gamma}{n^{2}}\right)^{\frac{\gamma}{n}}}{\left(1-\frac{\gamma}{n^{2}}\right)^{n+\frac{\gamma}{n}}}\left(\frac{1-\frac{\sqrt{\gamma}}{n}}{1+\frac{\sqrt{\gamma}}{n}}\right)^{2 \sqrt{\gamma}} .
$$

The equality in this inequality is achieved when $0, a_{k}$ and $B_{0}, B_{k}, k=\overline{1, n}$, are, respectively, poles and circular domains of the quadratic differential

$$
Q(w) d w^{2}=-\frac{\left(n^{2}-\gamma\right) w^{n}+\gamma}{w^{2}\left(w^{n}-1\right)^{2}} d w^{2} .
$$

The goal of this paper is to obtain some estimate of the functional $I_{n}(\gamma)$ for all $\gamma \in(1, n]$. Thus, we obtain the following statement.

Theorem 1. Let $n \in \mathbb{N}, n \geqslant 2, \gamma \in(1, n]$. Then, for any system of distinct points $\left\{a_{k}\right\}_{k=1}^{n}$ of the unit circle and any mutually non-overlapping domains $B_{k}, a_{k} \in B_{k} \subset \overline{\mathbb{C}}, k=\overline{0, n}$, $a_{0}=0$, the following inequality holds

$$
r^{\gamma}\left(B_{0}, 0\right) \prod_{k=1}^{n} r\left(B_{k}, a_{k}\right) \leqslant n^{-\frac{\gamma}{2}}\left(\prod_{k=1}^{n} r\left(B_{k}, a_{k}\right)\right)^{1-\frac{\gamma}{n}} .
$$

Proof. Let $\Delta$ be the maximum of the functional $I_{n}(\gamma)$. In papers $[1,4,8,9]$, the authors reviewed the case when $I_{n}(\gamma) \leqslant \Delta$. Consider the case if $\Delta \leqslant I_{n}(\gamma)$. Let $d(E)$ be the transfinite diameter of a compact set $E \subset \mathbb{C}$. Then the following relation holds

$$
r\left(B_{0}, 0\right)=r\left(B_{0}^{+}, \infty\right)=\frac{1}{d\left(\overline{\mathbb{C}} \backslash B_{0}^{+}\right)} \leqslant \frac{1}{d\left(\bigcup_{k=1}^{n} \bar{B}_{k}^{+}\right)},
$$

where $B^{+}=\left\{z: \frac{1}{z} \in B\right\}$. In view of the well-known Pólya theorem [6, p. 34], [18, p. 28], the inequality

$$
\mu E \leqslant \pi d^{2}(E)
$$


is valid, where $\mu E$ denotes the Lebesgue measure of a compact set $E$. Hence, we get

$$
d(E) \geqslant\left(\frac{1}{\pi} \mu E\right)^{\frac{1}{2}}
$$

Then relation (3) yields

$$
r\left(B_{0}, 0\right) \leqslant \frac{1}{d\left(\bigcup_{k=1}^{n} \bar{B}_{k}^{+}\right)} \leqslant \frac{1}{\sqrt{\frac{1}{\pi} \mu\left(\bigcup_{k=1}^{n} \bar{B}_{k}^{+}\right)}}=\left[\frac{1}{\pi} \sum_{k=1}^{n} \mu \bar{B}_{k}^{+}\right]^{-\frac{1}{2}} .
$$

Let $B$ be a bounded domain, $a \in B$. We consider the class of all regular functions $\varphi(z)$, given in the domain $B$ and such that $\varphi(a)=0, \varphi^{\prime}(a)=1$. Here we estimate the area of an image of the domain $B$ at the mapping by an arbitrary function $\varphi(z)$. It follows from the theorem of minimization of areas [6, p.34] that

$$
\iint_{B}\left|\varphi^{\prime}(z)\right|^{2} d x d y \geqslant \pi r^{2}(B, a) .
$$

Let us set $\varphi_{1}(z)=(z-a)$, then relation (5) yields

$$
S(B)=\mu(B) \geqslant \pi r^{2}(B, a) .
$$

Inequality (4) implies directly that

$$
r\left(B_{0}, 0\right) \leqslant\left[\frac{1}{\pi} \sum_{k=1}^{n} \mu \bar{B}_{k}^{+}\right]^{-\frac{1}{2}} \leqslant\left[\frac{1}{\pi} \sum_{k=1}^{n} \mu B_{k}^{+}\right]^{-\frac{1}{2}} \leqslant\left[\sum_{k=1}^{n} r^{2}\left(B_{k}^{+}, a_{k}^{+}\right)\right]^{-\frac{1}{2}} .
$$

Hence we get the inequality

$$
r\left(B_{0}, 0\right) \leqslant \frac{1}{\left[\sum_{k=1}^{n} r^{2}\left(B_{k}^{+}, a_{k}^{+}\right)\right]^{\frac{1}{2}}} .
$$

Taking into account the relation

$$
r\left(B_{k}^{+}, a_{k}^{+}\right)=\frac{r\left(B_{k}, a_{k}\right)}{\left|a_{k}\right|^{2}}
$$

we arrive at the inequality

$$
r\left(B_{0}, 0\right) \leqslant\left[1 / \sum_{k=1}^{n} \frac{r^{2}\left(B_{k}, a_{k}\right)}{\left|a_{k}\right|^{4}}\right]^{\frac{1}{2}} .
$$

This result and the assumption of Theorem 1 yield the relation

$$
\Delta \leqslant r^{\gamma}\left(B_{0}, 0\right) \prod_{k=1}^{n} r\left(B_{k}, a_{k}\right) \leqslant \frac{\prod_{k=1}^{n} r\left(B_{k}, a_{k}\right)}{\left[\sum_{k=1}^{n} \frac{r^{2}\left(B_{k}, a_{k}\right)}{\left|a_{k}\right|^{4}}\right]^{\frac{\gamma}{2}}}
$$


$\Delta$ is the maximum of functional $I_{n}(\gamma)$. The Cauchy inequality yields automatically the relation

And using the equality

$$
\frac{1}{n} \sum_{k=1}^{n} \frac{r^{2}\left(B_{k}, a_{k}\right)}{\left|a_{k}\right|^{4}} \geqslant\left[\prod_{k=1}^{n} \frac{r^{2}\left(B_{k}, a_{k}\right)}{\left|a_{k}\right|^{4}}\right]^{\frac{1}{n}}
$$

$$
\prod_{k=1}^{n}\left|a_{k}\right|=1
$$

we get easily

$$
\left[\sum_{k=1}^{n} \frac{r^{2}\left(B_{k}, a_{k}\right)}{\left|a_{k}\right|^{4}}\right]^{\frac{\gamma}{2}} \geqslant\left[n\left[\prod_{k=1}^{n} \frac{r^{2}\left(B_{k}, a_{k}\right)}{\left|a_{k}\right|^{4}}\right]^{\frac{1}{n}}\right]^{\frac{\gamma}{2}} \geqslant n^{\frac{\gamma}{2}}\left[\prod_{k=1}^{n} r\left(B_{k}, a_{k}\right)\right]^{\frac{\gamma}{n}} .
$$

Eventually,

$$
r^{\gamma}\left(B_{0}, 0\right) \prod_{k=1}^{n} r\left(B_{k}, a_{k}\right) \leqslant \frac{\prod_{k=1}^{n} r\left(B_{k}, a_{k}\right)}{n^{\frac{\gamma}{2}}\left[\prod_{k=1}^{n} r\left(B_{k}, a_{k}\right)\right]^{\frac{\gamma}{n}}}=n^{-\frac{\gamma}{2}}\left(\prod_{k=1}^{n} r\left(B_{k}, a_{k}\right)\right)^{1-\frac{\gamma}{n}} .
$$

Thus, Theorem 1 is proved.

Remark 1. If $\gamma=n$, then by Theorem 1 the following inequality holds

$$
r^{n}\left(B_{0}, 0\right) \prod_{k=1}^{n} r\left(B_{k}, a_{k}\right) \leqslant n^{-\frac{n}{2}}
$$

For any $n$-radial system of points $A_{n}=\left\{a_{k}\right\}_{k=1}^{n},\left|a_{k}\right|=1$, and for any pairwise nonoverlapping domains $\left\{B_{k}\right\}_{k=1}^{n}, a_{k} \in B_{k} \subset \overline{\mathbb{C}}, k=\overline{1, n}$, the inequality

$$
\prod_{k=1}^{n} r\left(B_{k}, a_{k}\right) \leqslant 2^{n} \prod_{k=1}^{n} \alpha_{k}
$$

is valid (see, [9, Corollary 5.1.3]). Using this result we obtain the following statement.

Corollary 1. Let $n \in \mathbb{N}, n \geqslant 2, \gamma \in(1, n]$. Then for any system of distinct points $\left\{a_{k}\right\}_{k=1}^{n}$ of the unit circle and any mutually non-overlapping domains $B_{k}, a_{k} \in B_{k} \subset \overline{\mathbb{C}}, k=\overline{0, n}$, $a_{0}=0$, the following inequality holds

$$
r^{\gamma}\left(B_{0}, 0\right) \prod_{k=1}^{n} r\left(B_{k}, a_{k}\right) \leqslant 2^{(n-\gamma)} \cdot n^{-\frac{\gamma}{2}} \cdot\left(\prod_{k=1}^{n} \alpha_{k}\right)^{1-\frac{\gamma}{n}} .
$$

In [8, Theorem 6.11] for any distinct points $a_{k}$ on the circle $\left|a_{k}\right|=1, k=\overline{1, n}(n \geqslant 2)$, and any pairwise non-overlapping domains $B_{k} \subset \overline{\mathbb{C}}$ such that $a_{k} \in B_{k}, k=\overline{1, n}$, the inequality

$$
\prod_{k=1}^{n} r\left(B_{k}, a_{k}\right) \leqslant\left(\frac{4}{n}\right)^{n}
$$

is proved. Thus, we have the next result. 
Corollary 2. Let $n \in \mathbb{N}, n \geqslant 2, \gamma \in(1, n]$. Then for any system of distinct points $\left\{a_{k}\right\}_{k=1}^{n}$ of the unit circle and any mutually non-overlapping domains $B_{k}, a_{k} \in B_{k} \subset \overline{\mathbb{C}}, k=\overline{0, n}$, $a_{0}=0$, the following inequality holds

$$
r^{\gamma}\left(B_{0}, 0\right) \prod_{k=1}^{n} r\left(B_{k}, a_{k}\right) \leqslant n^{-\frac{\gamma}{2}}\left(\frac{4}{n}\right)^{n-\gamma} .
$$

In view of Theorem 1 for any system of the distinct points $\left\{a_{k}\right\}_{k=1}^{n} \in \mathbb{C} \backslash\{0\}$ the following statement is true.

Corollary 3. Let $n \in \mathbb{N}, n \geqslant 2, \gamma \in(1, n]$. Then, for any system of distinct points $\left\{a_{k}\right\}_{k=1}^{n} \in \mathbb{C} \backslash\{0\}$ and any mutually non-overlapping domains $B_{k}, a_{k} \in B_{k} \subset \overline{\mathbb{C}}, k=\overline{0, n}$, $a_{0}=0$, the inequality

$$
r^{\gamma}\left(B_{0}, 0\right) \prod_{k=1}^{n} r\left(B_{k}, a_{k}\right) \leqslant n^{-\frac{\gamma}{2}}\left(\prod_{k=1}^{n} r\left(B_{k}, a_{k}\right)\right)^{1-\frac{\gamma}{n}}\left(\prod_{k=1}^{n}\left|a_{k}\right|\right)^{\frac{2 \gamma}{n}}
$$

holds.

The authors thank the anonymous referee for the very careful analysis of this work and remarks.

The publication contains the results of studies conducted by President's of Ukraine grant for competitive projects F75/30308 of the State Fund for Fundamental Research of Ukraine.

\section{REFERENCES}

1. Dubinin V.N., Symmetrization method in geometric function theory of complex variables, Uspekhy Matem. Nauk, 49 (1994), №1, 3-76. (in Russian); Engl.transl.: Russian Math. Surveys, 1 (1994), 179.

2. Dubinin V.N., The separating transformation of domains and problems on the extremal partition, Zap. Nauchn. Sem. LOMI, 168 (1988), 48-66. (in Russian)

3. Kuz'mina G.V., The method of extremal metric in extremal decomposition problems with free parameters, J. Math. Sci., 129 (2005), №3, 3843-3851.

4. Kovalev L.V., On the problem of extremal decomposition with free poles on a circle, Dal'nevostochnyi Mat. Sb., (1996), №2, 96-98. (in Russian)

5. Lavrent'ev M.A., On the theory of conformal mappings, Tr. Sci. Inst AN USSR, 5 (1934), 159-245. (in Russian)

6. Goluzin G.M., Geometric theory of functions of a complex variable, Amer. Math. Soc. Providence, R.I., 1969.

7. Jenkins J., Univalent functions and conformal mapping, Moscow: Publishing House of Foreign Literature, 256, 1962. (in Russian)

8. Dubinin V.N., Condenser capacities and symmetrization in geometric function theory, Birkhäuser/Springer, Basel, 2014.

9. Bakhtin A.K., Bakhtina G.P., Zelinskii Yu.B., Topological-algebraic structures and geometric methods in complex analysis, Zb. prats of the Inst. of Math. of NASU, 2008. (in Russian)

10. Bakhtin A.K., Denega I.V., Addendum to a theorem on extremal decomposition of the complex plane, Bulletin de la société des sciences et des lettres de Łódź, Recherches sur les déformations, 62 (2012), №2, 83-92. 
11. Denega I.V., Generalization of some extremal problems on non-overlapping domains with free poles, Annales universitatis Mariae Curie-Skladovska, LXVII (2013), №1, 11-22.

12. Zabolotnij Ja.V., Determination of the maximum of a product of inner radii of pairwise nonoverlapping domains, Dopov. Nats. Akad. Nauk Ukr., (2016), №3, 7-13. (in Ukrainian)

13. Bakhtin A., Dvorak I., Denega I., Separating transformation and extremal decomposition of the complex plane, Bulletin de la societe des sciences et des lettres de Lodz, Recherches sur les deformations, LXVI (2016), ㅅoㄴ, 13-20.

14. Bakhtin A., Vygivska L., Denega I., N-radial systems of points and problems for non-overlapping domains, Lobachevskii Journal of Mathematics, 38 (2017), №2, 229-235.

15. Bakhtin A.K., Zabolotnii Ya.V., Estimates of a product of the inner radii of nonoverlapping domains, J. Math. Sci., 221 (2017), №5, 623-629.

16. Bakhtin A.K., Vygivska L.V., Denega I.V., Inequalities for the internal radii of non-overlapping domains, J. Math. Sci., 220 (2017), №5, 584-590.

17. Denega I.V., Zabolotnii Ya.V., Estimates of products of inner radii of non-overlapping domains in the complex plane, Complex Variables and Elliptic Equations, 62 (2017), №11, 1611-1618.

18. Pólya G., Szegö G., Isoperimetric inequalities in mathematical physics, M.: Fizmatgiz, 1962.

Institute of Mathematics of National Academy of Sciences of Ukraine

Department of Complex analysis and Potential Theory

Kyiv, Ukraine

abahtin@imath.kiev.ua, iradenega@gmail.com 